\title{
Reagent Precoated Targets for Rapid In-Tissue Derivatization of the Anti-Tuberculosis Drug Isoniazid Followed by MALDI Imaging Mass Spectrometry
}

\author{
M. Lisa Manier, ${ }^{1}$ Michelle L. Reyzer, ${ }^{1}$ Anne Goh, ${ }^{2}$ Veronique Dartois,${ }^{2}$ Laura E. Via, ${ }^{3}$ \\ Clifton E. Barry III, ${ }^{3}$ Richard M. Caprioli ${ }^{1,4}$ \\ ${ }^{1}$ Mass Spectrometry Research Center, Vanderbilt University, Nashville, Tennessee, USA \\ ${ }^{2}$ Novartis Institute for Tropical Diseases, Biopolis, Singapore \\ ${ }^{3}$ National Institute of Allergy and Infectious Diseases, National Institutes of Health, Bethesda, Maryland, USA \\ ${ }^{4}$ Departments of Chemistry, Pharmacology, and Biochemistry, Vanderbilt University School of Medicine, 46521 st Avenue \\ South, Medical Research Building 3, Room 9160, Nashville, TN 37232-8575, USA
}

\begin{abstract}
Isoniazid (INH) is an important component of front-line anti-tuberculosis therapy with good serum pharmacokinetics but unknown ability to penetrate tuberculous lesions. However, endogenous background interferences hinder our ability to directly analyze INH in tissues. Chemical derivatization has been successfully used to measure isoniazid directly from tissue samples using matrix-assisted laser desorption/ionization (MALDI) imaging mass spectrometry (IMS). MALDI targets were pretreated with trans-cinnamaldehyde (CA) prior to mounting tissue slices. Isoniazid present in the tissues was efficiently derivatized and the INH-CA product measured by MS/MS. Precoating of MALDI targets allows the tissues to be directly thaw-mounted and derivatized, thus simplifying the preparation. A time-course series of tissues from tuberculosis infected/INH dosed animals were assayed and the MALDI MS/MS response correlates well with the amount of INH determined to be in the tissues by high-performance liquid chromatography (HPLC)-MS/MS.
\end{abstract}

Key words: Matrix-assisted laser desorption/ionization, Imaging mass spectrometry, Isoniazid, Tuberculosis, trans-cinnamaldehyde, Derivatization, Precoated MALDI targets

\section{Introduction}

ince the introduction of matrix-assisted laser desorption/ S ionization (MALDI)-based imaging mass spectrometry [1], this technology has been used to study the spatial localization of a variety of analytes directly from thin tissue sections, including proteins [2-4], peptides [5, 6], lipids [79], and small xenobiotics [10-12]. The advantages of imaging with MALDI mass spectrometry are numerous, including simple sample preparation, high sensitivity (atto-

Correspondence to: Richard Caprioli; e-mail: r.caprioli@vanderbilt.edu mole/femtomole range for many compounds), and relatively fast sampling speeds (up to $100 \mathrm{~ms} / \mathrm{pixel}$, depending on desired resolution and sensitivity). Imaging mass spectrometry (IMS) allows an assessment of local drug concentrations in situ within the lesions of infected animals and therefore adds highly valuable information to simple serum pharmacokinetic analyses.

While MALDI IMS is becoming increasingly popular for the analysis of drugs and metabolites within tissue sections, it can be challenging due to low molecular weight background signals. Endogenous species that may produce spectral interferences and/or contribute to ionization sup- 
pression are routinely removed from tissues using washing protocols prior to protein analysis [13-16] (for example, successive washes in graded ethanol solutions remove salts and most lipids); however, this approach may extract or delocalize small molecule analytes as well. Other approaches to overcome these interferences have involved the selective choice of MALDI matrix [17], the use of high-resolution FT-MS analysis [18], or MS/MS analysis [9, 11, 12].

In cases where compounds exhibit poor ionization efficiency, chemical derivatization may be employed to increase ion yields. Chemical derivatization $[19,20]$ is routinely used to improve detection of drugs by a variety of analytical techniques, including gas chromatography (GC), high-performance liquid chromatography (HPLC), and increasingly MALDI MS [21-30]. Recently, this approach has also been used in conjunction with IMS for direct tissue analysis [31]. For example, Franck et al. utilized the reactive ( $N$-succinimidyloxycarbonylmethyl)tris(2,4,6-trimethoxyphenyl) phosphonium bromide (TMPP) compound to direct peptide fragmentation towards the N-terminus for in situ tryptic peptides [31].

Adding a derivatization agent to tissue sections complicates the sample preparation process. The derivatization agent may contribute to analyte delocalization, signal suppression, and may interfere with matrix crystal formation. Precoating of MALDI targets has been employed as a strategy to apply matrix to a surface for improved reproducibility or sensitivity. Vorm et al. used fast evaporation of solvent from matrix solutions to produce a thin layer of homogenous microcrystals directly on a MALDI target plate [32]. Regenerated cellulose has been affixed to MALDI plates followed by treatment with $\alpha$-cyano-4-hydroxycinnamic acid (CHCA) in order to collect continuous effluent from a capillary electrophoresis unit [33]. Similarly, peptides from protein digests were resolved using capillary reversedphase HPLC and deposited onto CHCA-coated MALDI targets [34]. These precoated targets facilitated off-line coupling of HPLC separation with MALDI analysis, allowing improved sensitivity for low abundance peptides.

More recently, entire MALDI targets were precoated with 2,5-dihydroxybenzoic acid matrix using sublimation. Tissue sections were thaw-mounted onto the precoated targets and directly analyzed by MALDI IMS. This approach resulted in $1-2 \mu \mathrm{m}$ sized crystals with minimal analyte delocalization as no extraction solvents were applied. Lipids, peptides, and small molecules (drug compounds) were amenable to analysis using this technique [35].

Isoniazid (INH) is widely used in first-line treatment for tuberculosis as part of a four-drug combination therapy. INH is well absorbed and has good pharmacokinetic properties that result in a rapid bactericidal activity on organisms within patients' sputum [36, 37]. Unfortunately this activity is limited to the first month or two of treatment, after which it is thought to have little long-term "sterilizing" activity [38]. Sterilizing activity is a complex phenomenon related to the ability of the drug to prevent relapse with active disease after discontinuing chemotherapy. The poor sterilizing activity of the four drug combination is reflected in the 68 mo regimen required to achieve a sterile cure. There are many different theories around the intrinsic sterilizing potential of various drugs [39] but perhaps the most straightforward is related to the ability of drugs to partition out of serum and into the dense granulomas that form the basis of much of the lung pathology of the disease. To test that theory, we have been exploring IMS as a method to assess the tissue penetration of TB drugs into lesions. Our initial efforts to study INH distribution within tissue sections by MALDI IMS proved difficult due to endogenous tissue interferences. Isoniazid has been derivatized previously using trans-cinnamaldehyde [40] and $N$-methyl-9-chloroacridinium triflate [41] to improve its detection by HPLC with ultraviolet/visible (UV/Vis) analysis. It has also been analyzed as the copper chelated Schiff base of salicylaldehyde-5-sulfonate using capillary zone electrophoresis [42].

Here we present an in-tissue chemical derivatization protocol for isoniazid in order to attain suitable sensitivity for imaging analyses of dosed tissue samples using MALDI IMS. We investigated the derivatization of isoniazid under various $\mathrm{pH}$, temperature, time, and solvent conditions using several aldehydes, steroids, and succinimidyl carbamates. The optimal reagent, trans-cinnamaldehyde, was then applied to MALDI targets prior to tissue samples and utilized for the analysis of lung tissue sections from tuberculosis infected rabbits treated with a multi-drug mixture containing isoniazid. The reagent precoated targets allow for rapid in-tissue derivatization and thus provide a convenient platform for imaging MS studies where derivatization is required.

\section{Experimental}

\section{Materials}

The following reagents were purchased from Sigma-Aldrich Chemical Co. (St. Louis, MO, USA) and used for INH derivatization: trans-cinnamaldehyde, $\beta$-phenylcinnamaldehyde, $\alpha$-bromocinnamaldehyde, 4-hydroxy-3-methoxycinnamaldehyde, 4-nitrocinnamaldehyde, trans-4-(diethylamino) cinnamaldehyde, hydrocortisone, and ( $N$-succinimidyloxycarbonylmethyl)tris(2,4,6-trimethoxyphenyl)phosphonium bromide (TMPP). Additionally, Waters AccQ-Fluor derivatizing reagent (6-aminoquinolyl- $N$-hydroxysuccinimidyl carbamate) was purchased from Waters Corporation (Milford, MA, USA). Methanol and acetonitrile (both HPLC grade) were obtained from Fisher Scientific (Pittsburgh, PA). Trifluoroacetic acid (TFA), isoniazid and $\alpha$-cyano-4-hydroxycinnamic acid (CHCA) were also obtained from Sigma-Aldrich.

\section{Method Development with Derivatization Reagents for MALDI IMS Analysis}

Chemical derivatives of isoniazid were formed with the nine different reagents. Parameters were based on those recom- 
mended in published methods for derivative reactions of similar compounds $[19,20,31,40,43-45]$. For tissue analysis, derivatization reagents were applied by either spray coating, sublimation, or spin coating (detailed below). Based on preliminary data, trans-cinnamaldehyde (CA) was chosen for analysis of study samples.

\section{Animal Studies}

Experiments utilizing New Zealand White (NZW) rabbits aerosol infected with $M$. tuberculosis as previously described [46] were performed with the approval of the animal care and use committee (ACUC) of NIH/NIAID under assurance \#A-4149-01 and protocol \#LCID-3. Tuberculosis-infected rabbits were dosed with rifampin/isoniazid/ pyrazinamide/moxifloxacin at 30/50/125/25 mg/ $\mathrm{kg}$ administered orally once daily for $1 \mathrm{wk}$, then sacrificed at various time points after the final dose. Control rabbits were dosed with rifampin $(24 \mathrm{mg} / \mathrm{kg})$. For MALDI analyses, lung regions containing lesions were snap-frozen in vapor phase liquid nitrogen, subjected to $3 \mathrm{Mrad} \gamma$ radiation as a sterilization procedure, shipped frozen on dry ice, and stored at $-80{ }^{\circ} \mathrm{C}$ until use.

\section{Tissue Preparation for HPLC-MS/MS Analysis}

Drug containing tissues were processed at the time of sacrifice (BSL-3 facility) with the sample preparation procedure described below, and the final lyophilized extracts were shipped for quantitative analysis. This preparation procedure was validated to sterilize the samples prior to shipment. Tissue was homogenized in phosphate buffered saline (PBS) using a Qiagen TissueLyser bead beater (Hilden, North Rhine-Westphalia, Germany), with $1 \mathrm{~mL}$ PBS added for every $0.2 \mathrm{~g}$ of tissue. Warfarin was added as an internal standard. Proteins were precipitated and $\mathrm{INH}$ extracted by addition of acetonitrile with $0.2 \%$ acetic acid in a 9:1 ratio of extractant to tissue homogenate. Samples were vortexed for $1 \mathrm{~min}$, centrifuged at $1000 \mathrm{~g}$ for $10 \mathrm{~min}$, and the supernatant removed. The extracts were lyophilized, shipped on dry ice, and frozen at $-20{ }^{\circ} \mathrm{C}$ until they were reconstituted in mobile phase and analyzed. Drug free tissue that was infected with $M$. tuberculosis and sterilized via $\gamma$ irradiation was used as naive material for calibration standards, quality control samples and blanks. Each was spiked with warfarin internal standard, then processed by the procedure used for study samples. Calibration standards ranged from $10.2 \mathrm{ng} / \mathrm{mL}$ to $10.4 \mu \mathrm{g} / \mathrm{mL}$.

\section{Tissue Preparation for MALDI IMS}

The frozen tissues were cut at $-20{ }^{\circ} \mathrm{C}$ into $12-\mu \mathrm{m}$ thick sections on a cryostat (Leica Microsystems Inc., Bannockburn, IL, USA) and thaw-mounted directly onto gold-coated stainless-steel MALDI target plates (Applied Biosystems, Foster City, CA, USA) or onto target plates precoated with trans-cinnamaldehyde. Tissues were analyzed by two different methods: (1) for underivatized INH or (2) for INH derivatized with CA using precoated targets as detailed below. In addition, a serial section of each tissue was cut and mounted on a glass slide and stained with hematoxylin and eosin (H\&E) for anatomical visualization.

CHCA was used as the MALDI matrix and was prepared as a $15 \mathrm{mg} / \mathrm{mL}$ solution in $60: 40$ acetonitrile:water (with $0.1 \%$ TFA). CHCA was coated over the entire surface of all tissues mounted on MALDI plates. This was accomplished by placing the solution into a thin layer chromatography (TLC) glass reagent sprayer (Kontes Glass Company, Vineland, NJ, USA) and spraying multiple coats at a distance of $\sim 20 \mathrm{~cm}$ across the surface of the tissue. One coating cycle consisted of passing the sprayer two times across the surface of the tissue and allowing the tissue to dry. This process was repeated until there was a homogeneous layer of matrix crystals over the surface of the tissue, usually about 5-8 cycles. The plates were desiccated for at least $15 \mathrm{~min}$ prior to instrumental analysis.

\section{Pretreatment of MALDI Plates with CA Using High Velocity Spin Coating}

In order to create precoated targets, trans-cinnamaldehyde was deposited on MALDI target plates using high velocity spin coating. A target was vacuum sealed to the chuck of a WS-400 bench-top single wafer spinner (Laurell Technologies Corporation, North Wales, PA, USA). Approximately $1 \mathrm{~mL}$ of $50 \%$ $\mathrm{CA}$ in methanol was deposited onto the center of the plate and spinning commenced for $10 \mathrm{~s}$ at $500 \mathrm{rpm}$ for an initial spread of reagent. The speed was then increased to $2000 \mathrm{rpm}$ for $20 \mathrm{~s}$ to form a homogeneous coating of CA. The CA-coated plate was stored overnight in the dark at room temperature to allow crystal formation prior to tissue placement the following day. After tissues were thaw-mounted, the plates were allowed to incubate for at least $30 \mathrm{~min}$ at room temperature prior to matrix application as detailed above.

\section{HPLC-MS/MS Analysis}

Lyophilized extracts were thawed and reconstituted into 200 $\mu \mathrm{L}$ mobile phase. Ten $\mu \mathrm{L}$ were injected into an HPLC system (Symbiosis Pharma, Spark Systems Solutions, The Netherlands) and separated at a flow rate of $1 \mathrm{~mL} / \mathrm{min}$ on a Gemini C6-phenyl $4.6 \times 150 \mathrm{~mm}, 5 \mu \mathrm{m}$ column (Phenomenex, Torrance, California, USA) held at $35{ }^{\circ} \mathrm{C}$. A gradient elution was used, starting with $97 \%$ mobile phase A (water with $0.2 \%$ acetic acid) and going to $85 \%$ mobile phase B (methanol with $0.2 \%$ acetic acid) in $4.5 \mathrm{~min}$. The analysis was performed using an Applied Biosystems/Sciex API4000 triple-quadrupole mass spectrometer (Foster City, California, USA). Selected reaction monitoring (SRM) of precursor-toproduct transitions were used in electrospray positive ionization mode to track presence of analytes. Quantitation of drug levels was performed using the $\mathrm{m} / \mathrm{z}$ transitions 
$309.2 \rightarrow 251.1$ for warfarin and $138.0 \rightarrow 121.1$ for isoniazid with collision energies of 28 and $21 \mathrm{eV}$, respectively. Although rifampin, pyrazinamide, and moxifloxacin were quantified also, results are not presented here. The flow rates of curtain and collision gas were set to 25 and 6 psi, respectively. Ultra pure nitrogen was used with a nebulizing temperature of $500{ }^{\circ} \mathrm{C}$. The ion spray voltage, nebulizing gas (GS1) and Turbo gas (GS2) were set at $5500 \mathrm{~V}, 45$ and 60 psi, respectively. The dwell time for each SRM transition was $100 \mathrm{~ms}$. Compound specific MS parameters were optimized for each analyte. Calibration curve data were evaluated using a quadratic fit and $1 / \mathrm{x}$ weighting. Sample analysis was accepted if the low level quality control samples were within $\pm 20 \%$ of nominal concentration and $\pm 15 \%$ for mid and high level quality control samples.

\section{MALDI Imaging Mass Spectrometry}

Mass spectra were acquired in positive-ion mode using a MALDI LTQ XL linear ion trap mass spectrometer (Thermo

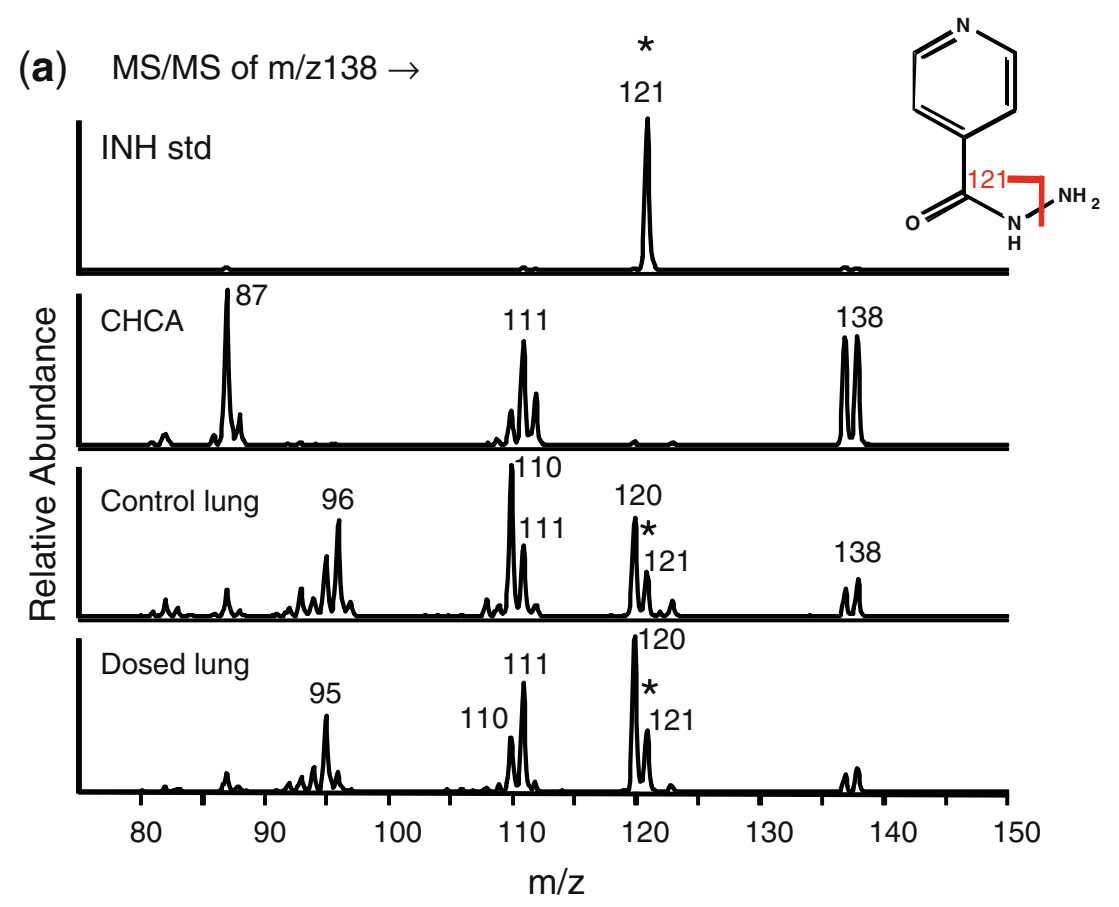

(b)
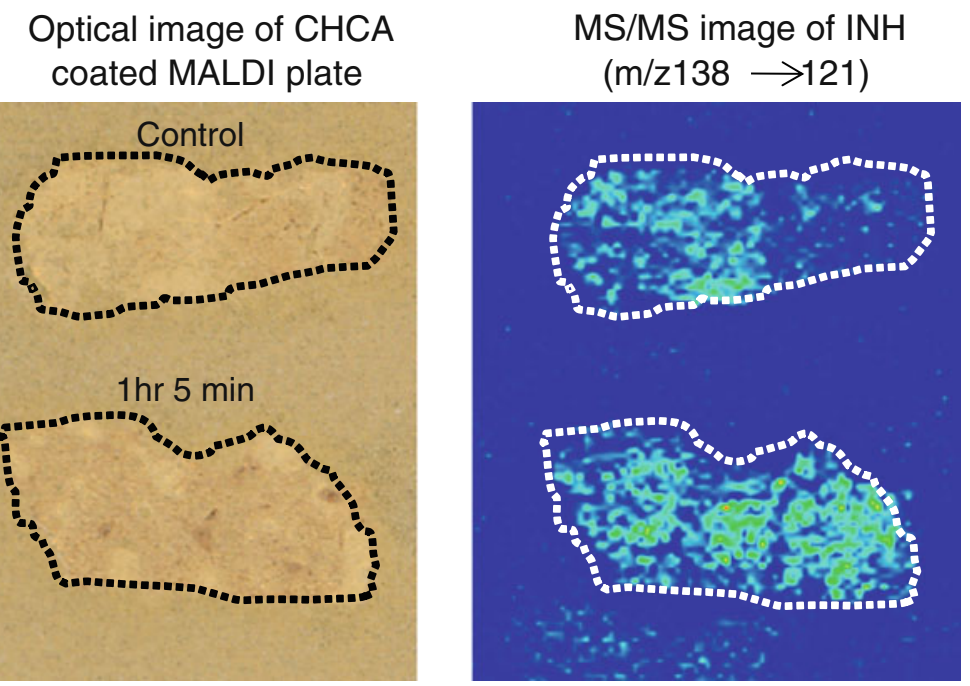

Figure 1. Analysis of underivatized INH in rabbit lung tissue. The animal was dosed with rifampin/isoniazid/pyrazinamide/ moxifloxacin at 30/50/125/25 mg/kg/d for $1 \mathrm{wk}$ and sacrificed $1 \mathrm{~h} 5 \mathrm{~min}$ after the final dose. Non-INH dosed control tissue was also analyzed. (a) MS/MS spectra for INH $(\mathrm{m} / z$ 138 $\rightarrow$ ) from prepared standard (250 pmol on plate), CHCA blank, control lung, and dosed lung. (b) Optical image of rabbit tissue sections coated with $\mathrm{CHCA}$ (left) and reconstructed ion image of INH in rabbit tissues (right). The transition $(m / z 138 \rightarrow 121)$ was monitored. Due to endogenous background, it is not possible to distinguish dosed from control rabbit lung 
Scientific, San Jose, CA, USA), equipped with a $337-\mathrm{nm}$ nitrogen laser operating at $60 \mathrm{~Hz}$ and resulting in $\sim 40 \times 100$ $\mu \mathrm{m}$ laser spot size. Dissociation of protonated molecules was achieved using an isolation window of $1 \mathrm{Da}$ and CID energy of 40 for INH or 50 for INH-CA (arbitrary units) with helium as the trapping/collision gas. Isolated precursor ions were activated for $30 \mathrm{~ms}$ using an $\mathrm{rf}$ frequency activation $\mathrm{Q}$ value of 0.5 (INH) or 0.25 (INH-CA). Product ion mass spectra were acquired over the $\mathrm{m} / \mathrm{z}$ range $75-170$ (INH) and 65-300 (INH-CA). For MS/MS imaging experiments, an average of 15 laser shots per scan was used to produce a mass spectrum every $200 \mu \mathrm{m}$ laterally across the tissue. Two dimensional ion density images were extracted from the raw data using ImageQuest ver. 1.0.1 (Thermo Scientific, San Jose, CA, USA).

\section{Results and Discussion}

\section{Imaging MS/MS Analysis of Underivatized INH}

Analysis of INH by mass spectrometry is straightforward using high-performance liquid chromatography and electrospray ionization [47-51]. However, due to endogenous interfering components at this very low mass range in tissue, the in situ analysis of INH by MALDI has been challenging. Fragmentation of underivatized INH yields predominantly one product ion $(\mathrm{m} / z 138 \rightarrow 121)$ as shown for the standard in Figure 1a (top spectrum). Unfortunately, there is also an endogenous background signal in rabbit lung tissue with the same transition (Figure 1a, control lung spectrum). Due to high background at $\mathrm{m} / \mathrm{z} 121$ in the lung tissue, it is not possible to easily distinguish control from dosed ( $1 \mathrm{~h} 5 \mathrm{~min}$ ) tissue (Figure 1b), even though this tissue contains a relatively high concentration of INH (approximately $10 \mu \mathrm{g} /$ $\mathrm{g}$ tissue, as determined by HPLC-MS/MS). MS $^{3}$ capabilities of the ion trap $(\mathrm{m} / \mathrm{z} \quad 138 \rightarrow 121 \rightarrow)$ were also utilized but did not improve the results. Therefore, derivatization of INH in lung tissue was investigated.

\section{Chemical Derivatization of INH}

We explored derivatives of INH in solution with each of the reagents listed in Table 1 under various solvent, $\mathrm{pH}$, concentration, temperature, and time conditions following recommended published methods for similar derivative reactions [19, 20, 31, 40, 43-45]. Listed are INH-derivative precursor ions $\left([\mathrm{M}]^{+}\right.$or $\left.[\mathrm{M}+\mathrm{H}]^{+}\right)$, predominant MS/MS fragments, derivative-to-INH molar ratios, and reaction parameters. Under the conditions used, some reagents formed derivatives in solution virtually immediately at room temperature (e.g., trans-cinnamaldehyde) while others were more easily formed at higher temperatures and elevated $\mathrm{pH}$ conditions (TMPP). The most promising derivatization agents were further evaluated on tissue sections and were applied by spray or spin coating or by sublimation [7] as described previously.

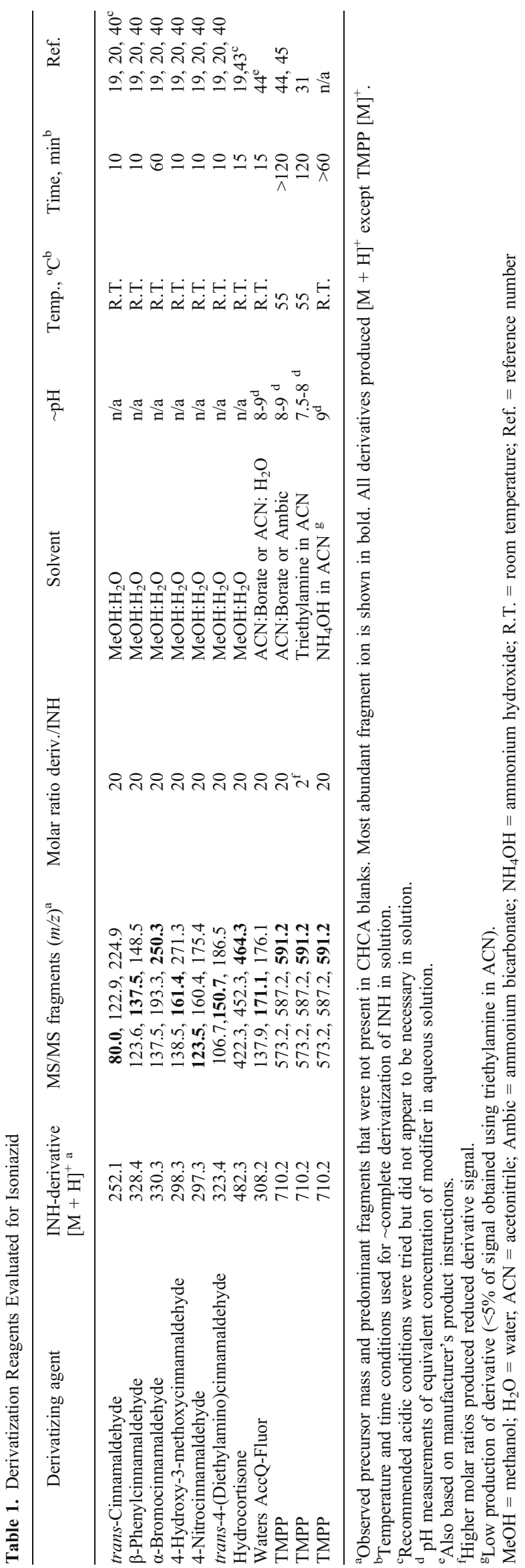


Sublimation is an obvious choice to precoat reagent on targets because it forms a very homogeneous coating in a relatively short period of time $(5-15 \mathrm{~min})$. While transcinnamaldehyde provided excellent sensitivity in derivatizing $\mathrm{INH}$, it is a liquid and thus not amenable to sublimation. In addition, MALDI plates coated with CA via spin coating required overnight drying prior to use. Thus, other cinnamaldehyde-based derivatization agents, which are solids at room temperature, were evaluated including $\beta$-phenylcinnamaldehyde and 4-hydroxy-3-methoxycinnamaldehyde (Methods section and Table 1). These reagents were precoated onto MALDI targets using sublimation procedures. Uniform solid coatings of reagents were obtained; however derivatization of INH in tissue samples did not occur on the precoated target alone in the absence of added solvent. Thus, the target plates were placed in a chamber containing methanol and acetonitrile vapor at room temperature for $10 \mathrm{~min}$ to $1 \mathrm{~h}$ in order to wet the tissues and promote INH-derivative formation. Plates were then coated with CHCA matrix. The reproducibility of the INHderivative signal was not reliable plate-to-plate and, in some instances, no significant signal was obtained above undosed tissue background; therefore no further work was done with these reagents.

Hydrocortisone produced an INH derivative $\left([\mathrm{M}+\mathrm{H}]^{+}=\right.$ 482) with standard INH, however it was not further evaluated in tissue. The major MS/MS fragments of the INH-hydrocortisone derivative are $\mathrm{m} / z 464$ and 452, corresponding to losses of $\mathrm{H}_{2} \mathrm{O}$ and $\mathrm{CH}_{2} \mathrm{O}$, respectively. These are not very specific losses and therefore potentially more susceptible to endogenous interferences. Two succinimidyl carbamates were studied, Waters AccQ-Fluor and TMPP, which both formed detectable derivatives with INH in solution. Waters AccQ-Fluor was not evaluated in tissue because the amount of reagent required to coat a target plate was cost-prohibitive. The other, TMPP, forms a derivative with INH that is positively charged and thus readily detected by MS. TMPP was further evaluated with several different modifiers (Table 1) to obtain $\mathrm{pH} \sim 8-9$, which is optimal for derivatization. Better sensitivity was obtained using triethylamine (TEA) versus ammonium hydroxide, borate, or ammonium bicarbonate buffers. Derivatization of INH in tissues using TMPP did not appear as promising as transcinnamaldehyde, although extensive optimization was not performed. trans-Cinnamaldehyde was used for derivatization of INH in subsequent tissue analysis because of its ease of use and improved sensitivity compared with the other derivatization reagents.

The structures of INH, CA, and the INH-CA product are shown in Scheme 1. The carbonyl of cinnamaldehyde forms a stable Schiff base with the hydrazide moiety of INH resulting in an INH-CA compound of $[\mathrm{M}+\mathrm{H}]^{+}=252.1$. This derivative forms quickly; CA vapor from INH-CA standards spotted on MALDI plates readily derivatized INH in nearby target wells. MS/MS of the derivative at $\mathrm{m} / \mathrm{z} 252$ produces three main fragment ions at $\mathrm{m} / z 225,123$, and 80 as shown in Figure 2a. No significant signals were observed from the matrix or cinnamaldehyde itself at these transitions (Figure 2b). Thus, chemical background from these compounds is quite low.

\section{Reagent Precoated Targets}

CA was initially applied to tissues by two techniques. In one, tissues mounted on MALDI target plates were spraycoated with a CA solution $(10 \mathrm{mM})$ using a TLC reagent sprayer. In this case, the CA contacted the tissue as a liquid, and several coatings were applied over time. In the second method, CA was applied to MALDI target plates (via a spincoating technique), allowed to dry, and tissue sections were subsequently thaw-mounted on the CA-coated target. Although $\mathrm{CA}$ is a liquid at room temperature, crystals formed on the target plates, likely due to partial oxidation to trans-cinnamic acid [52]. Presumably enough unoxidized $\mathrm{CA}$ remains in the crystals to react with the $\mathrm{INH}$, as the oxidized form would not be expected to form the derivative. Nuclear magnetic resonance (NMR) analysis of a dried coating indicated that approximately 1:1 CA:cinnamic acid was present. Subsequent studies have shown that a mixture of $\sim 1: 1$ trans-cinnamaldehyde:trans-cinnamic acid may be directly applied to MALDI target plates. These plates readily produce uniform coatings without overnight drying/oxidation. In addition, this mixture has been applied by spray coating either manually using an artist airbrush or automatically using a robotic sprayer (TM Sprayer; HTX Technologies, LLC, Carrboro, NC, USA) with excellent results.

Tissues applied to plates spin-coated with CA generally gave $\sim 10$-fold more INH-CA signal than tissues that were

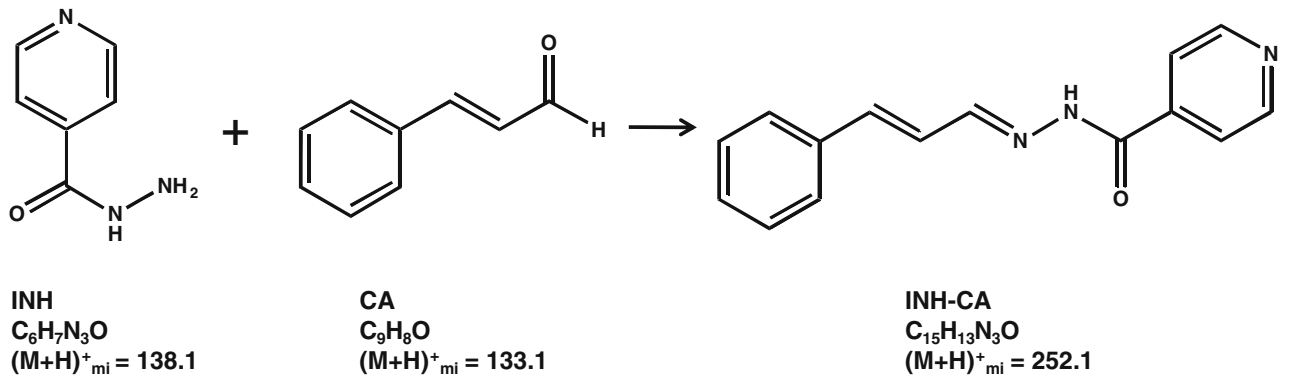

Scheme 1. Structures of INH, CA, and INH-CA with monoisotopic molecular weights of protonated molecules 


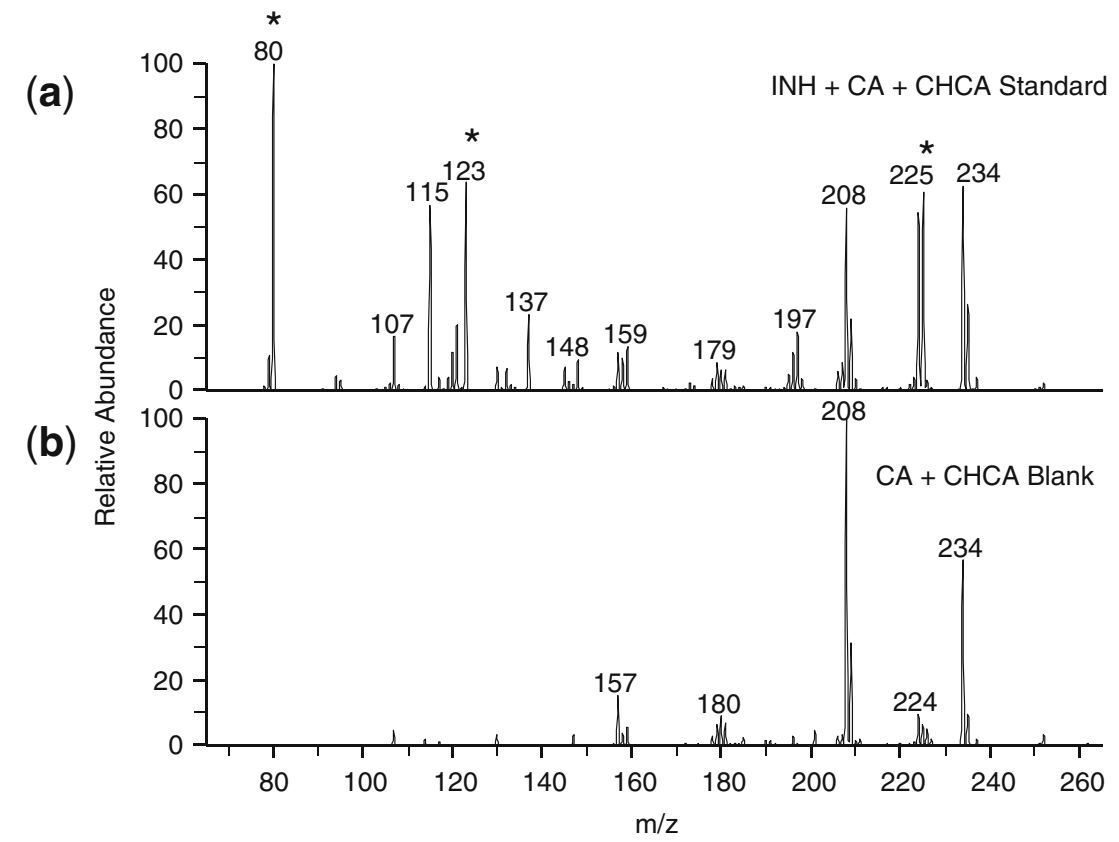

Figure 2. MS/MS spectra for INH-CA derivative $(m / z 252 \rightarrow)$ from (a) prepared standard (5 pmol on plate) (b) $\mathrm{CA}+\mathrm{CHCA}$ blank

spray-coated with the derivatization agent. While it is difficult to quantify the absolute amount of trans-cinnamaldehyde deposited onto the tissues with either method, based on the weight gained per plate following $\mathrm{CA}$ deposition (approximately $0.06 \mathrm{mg}$ for spray coating and $60 \mathrm{mg}$ for spin coating), there is approximately $1000 \times$ more CA present on a precoated target, thus providing a greater molar excess of CA compared to $\mathrm{INH}$.

In order to more accurately determine the molar ratio of CA needed to effectively derivatize INH in tissues, various amounts of CA (50 to 5,000,000 pmol) were applied in discrete areas on dosed tissue sections (calculated to contain $\sim 10 \mu \mathrm{g} \mathrm{INH} / \mathrm{g}$ tissue or $70 \mathrm{pmol} \mathrm{INH} / \mathrm{mg}$ tissue). CHCA matrix was applied on top of the CA spots. All spots were evaluated for the INH-CA derivative $(\mathrm{m} / \mathrm{z} 252 \rightarrow 80)$.

Approximately 500,000 pmol is required for maximum signal (Figure 3). This corresponds to a molar ratio of roughly 250,000:1 (assuming the tissue section is $1 \mathrm{mg}$ and roughly $8 \times 15 \mathrm{~mm}=120 \mathrm{~mm}^{2}$ and the area under the matrix spot is $3.14 \mathrm{~mm}^{2}$ resulting in $\sim 2 \mathrm{pmol} \mathrm{INH} / \mathrm{spot}$ ). At higher molar ratios, the INH-CA signals are lower, possibly because the increased amount of $\mathrm{CA}$ alters crystallization of CHCA or causes signal suppression. For premixed solutions a molar ratio of $\mathrm{CA}$ :INH of $\sim 100$ appears to be optimum (data not shown) suggesting that tissue contains compounds that may react or interfere with $\mathrm{CA}$ derivatization of INH. Based on these results, it is likely the precoated targets contain enough CA to successfully derivatize the INH present in tissues from dosed animals.

Due to the lack of sufficient INH-dosed lung tissue, we were unable to conduct additional studies of tissue thickness versus derivatization. Studies using rabbit liver $(3,6,12 \mu \mathrm{m})$ showed relative INH-CA intensities of $2,1.5$, 1 , respec- tively, indicating better derivatization with thinner tissue. However, it is difficult to section unembedded lung tissues thinly without extensive tearing and folding. Thus, all lung tissues were sectioned at $12 \mu \mathrm{m}$, but because all lung tissues in this study were processed identically, direct comparison among these tissues is reasonable.

\section{Imaging MS/MS Analysis of INH-CA in Rabbit Lung Tissues}

A series of lung tissues from rabbits dosed with INH and sacrificed at various time points, as well as two non-dosed

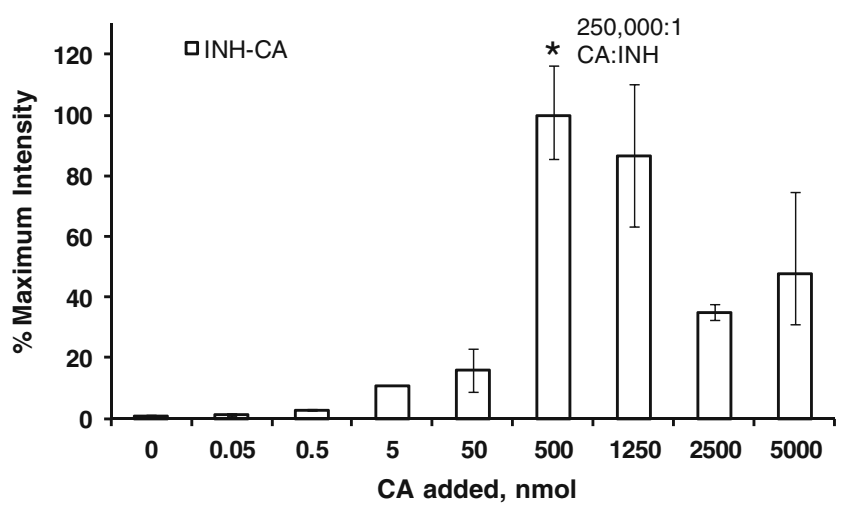

Figure 3. The effect of CA:INH ratio on in situ derivatization of INH. Various amounts of CA (from 0 to $5000 \mathrm{nmol} ; n=2-4$ ) were added to dosed tissue calculated to contain $\sim 70 \mathrm{pmol}$ $\mathrm{INH} / \mathrm{mg}$ tissue and the resulting CA derivative $(\mathrm{m} / \mathrm{z} \quad 252 \rightarrow 80)$ was monitored. The optimum molar ratio was found to be $\sim 250,000: 1$. Error bars indicate the highest and lowest values obtained, however, they are too small to be observed for values of $0-5 \mathrm{nmol}$ 
(a)

MS/MS of $\mathrm{m} / \mathrm{z} 252 \rightarrow$

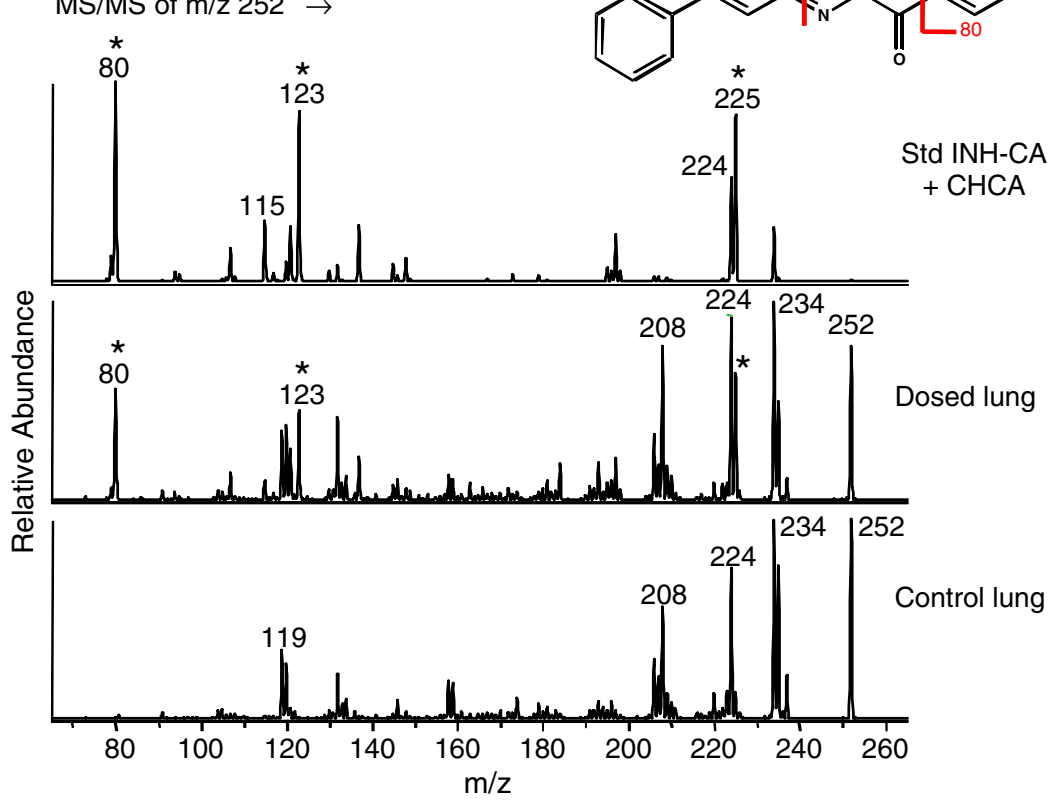

(b)

Optical image of tissue placed on $\mathrm{CA}$ spin-coated plate $+\mathrm{CHCA}$

$\mathrm{MS} / \mathrm{MS}$ image of INH-CA

$(\mathrm{m} / \mathrm{z} 252 \rightarrow 80 / \mathrm{TIC})$
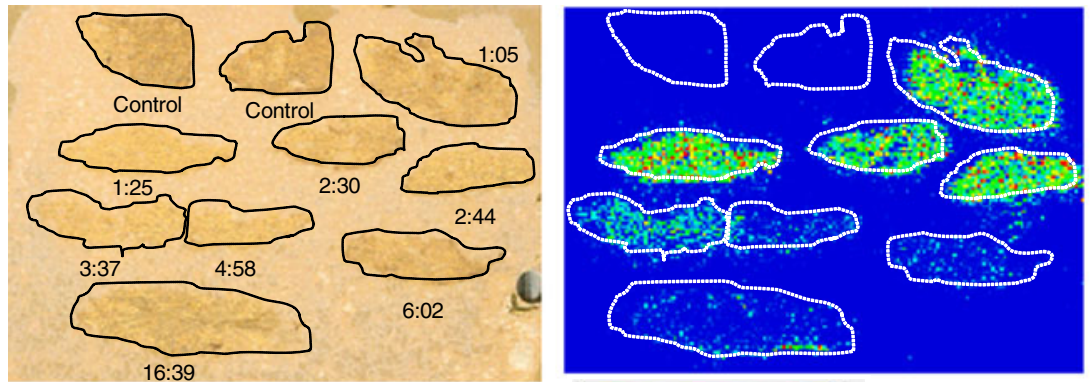

1.00 2.003.004.005.00 6.007.00

(c)
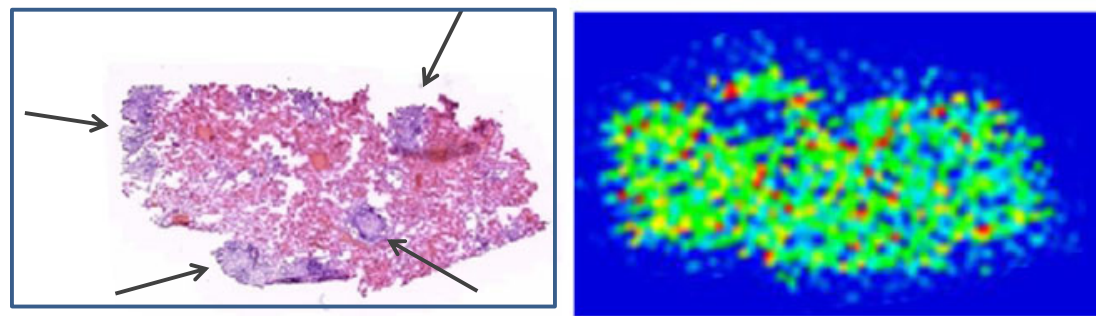

Figure 4. Analysis of INH-CA derivative in rabbit lung tissue. Animals were dosed with rifampin/isoniazid/pyrazinamide/ moxifloxacin at 30/50/125/25 mg/kg/d for $1 \mathrm{wk}$ and sacrificed at various h:min after final dose. Non-INH dosed control tissues from two different rabbits were also analyzed. (a) MS/MS spectra for INH-CA derivative $(\mathrm{m} / z$ 252 $\rightarrow)$ from prepared standard (125 pmol on plate), dosed lung from the image below (1 h 25 min), and control lung. (b) Optical image and MALDI image of rabbit tissues. Tissues were placed on targets that had been precoated with a layer of CA followed by CHCA deposition. The transition ( $\mathrm{m} / \mathrm{z} 252 \rightarrow 80 /$ total ion current) was used for ion image reconstruction. (c) H\&E stained serial section for lung tissue from the $1 \mathrm{~h} 5 \mathrm{~min}$ rabbit and expanded view of the MALDI image from (b). Lesions apparent as nuclei-rich dark purple areas (H\&E) are indicated by arrows. This protocol allowed visualization of INH in dosed tissues up to $\sim 17 \mathrm{~h}$, with minimal interfering signal from the control tissue, however no localization to unaffected lung or lesion areas is observed 
control tissues, were thaw-mounted onto a target plate that was precoated with CA as described. CHCA matrix was applied and the tissues were analyzed via MS/MS of $m / z 252$. Data were acquired as one file over all of the tissue sections. There is virtually no endogenous background in control tissue at the three major fragment masses $(\mathrm{m} / \mathrm{z}$ 225, 123, and 80) as shown in Figure 4a. The transition $\mathrm{m} / \mathrm{z} 252 \rightarrow 80$ was chosen for image reconstruction, however, reconstructed ion images for $\mathrm{m} / \mathrm{z} 123$ and 225 were similar. As shown in Figure 4b, INH is detected in all of the dosed rabbit tissues up to $\sim 17 \mathrm{~h}$ post-dose, while no significant signal is observed in the non-dosed control lung tissue. The time-course clearly shows an accumulation of INH at the early time points followed by a reduction of signal with time as the drug is cleared from the body. The $\mathrm{T}_{\max }$ (time to maximum serum concentration) for INH has been found to be $1 \mathrm{~h}$ or less and the $t_{1 / 2}$ (the half-life or rate of clearance) is $1 \mathrm{~h}$ to $1 \mathrm{~h} 30 \mathrm{~min}$ in rabbits (Via, Dartois, unpublished).

No significant localization of INH to either the unaffected lung tissue or lesion area was observed. For example, Figure $4 \mathrm{c}$ shows the H\&E stained serial section for lung tissue from the $1 \mathrm{~h} 5 \mathrm{~min}$ rabbit as well as an expanded view of the MALDI ion image for the section analyzed in Figure $4 \mathrm{~b}$. Lesions are shown as dark purple regions (indicated by arrows) and the center one measures $>1000$ $\mu \mathrm{m}$. As shown in the MALDI image, the INH-CA product is uniformly distributed over the tissue and no observable localization either to visually normal lung or granuloma is observed. The uniform distribution is not likely a result of any analyte delocalization; no significant INH-CA signal appears on the MALDI target outside of the tissue borders. However, the tissues evaluated in this study did not have large granulomas, therefore a more thorough assessment may be necessary using tissues from animals dosed with high concentrations of INH that also contain a number of larger lesions.

\section{Correlation of MALDI MS/MS and HPLC-MS/MS Data}

Absolute quantitation of INH in the tissue sections was not attempted via MALDI MS. The goal of the current study was to detect INH in tissue sections (via derivatization in this case) by IMS and evaluate localization of drug in unaffected lung tissue compared to $\mathrm{TB}$ lesion areas. However, it is reasonable to expect the signal response to be a function of drug concentration $[11,53]$. Therefore, the MALDI LTQ response for INH-CA from the current rabbit study was compared with the results obtained by HPLC-MS/ MS. The comparison is shown in Figure 5. Each time point represents a different animal that was evaluated by both MALDI and HPLC-MS/MS. The concentration of INH in unaffected lung tissue as determined by HPLC-MS/MS is expressed as $\mathrm{ng} \mathrm{INH} / \mathrm{g}$ tissue while the MALDI results are INH-CA signal intensities $(m / z 252 \rightarrow 80)$ from tissues

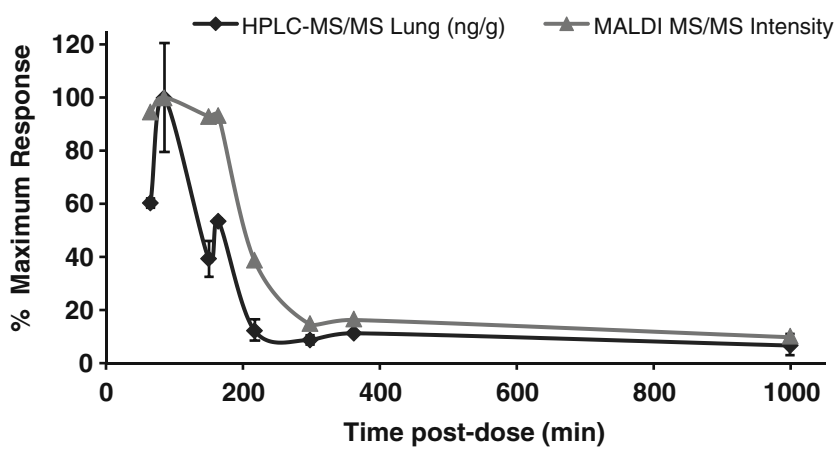

Figure 5. Correlation of MALDI MS/MS and HPLC-MS/MS data. MALDI signal intensities $(\mathrm{m} / \mathrm{z} 252 \rightarrow 80)$ were obtained by averaging the intensity (150 scans) over each tissue shown in Figure 4, while HPLC-MS/MS results are an average of multiple areas of lung $(n=2-4)$. For direct comparison, each value was corrected to \% of maximum response. HPLC-MS/ MS variability noted by error bars $( \pm 1 \sigma)$ while MALDI data is from a single analysis. A similar trend is observed for both HPLC-MS/MS and MALDI MS/MS

shown in Figure 4 (average of 150 scans from each tissue section). For direct comparison, both values were converted into percent of maximum response. MALDI data were obtained from tissue located in only one area of the lung (for example, right upper lobe) while the HPLC data are averages of multiple lung areas $(n=2-4)$ and not always from the same area as that taken for MALDI analysis. Therefore, some variability is expected (calculated \%RSDs range from 3 to 70 for HPLC lung results). Excised granulomas were also analyzed by HPLC-MS/MS for INH, and showed a similar trend (data not shown). Overall, no major differences in lung versus lesion concentrations of INH are noted with either HPLC-MS/MS or MALDI IMS. However, data do show that as the amount of drug in tissue decreases (as determined by HPLC-MS/MS) the MALDI MS/MS signal decreases; thus the trends observed for the two techniques are largely similar.

\section{Conclusions}

In-tissue derivatization has been shown to improve the detection of INH in cases where the analysis is hindered by isobaric interferences. High-resolution analysis may be used to distinguish isobaric species, however, this capability is not always available and may not improve the analysis if sensitivity is also an issue. For these instances, chemical derivatization is a viable alternative. For low molecular weight analytes, derivatization should ideally shift the molecular weight into a less complex spectral region (e.g., away from MALDI matrix ions and low mass contaminants). Sensitivity may be enhanced by using a derivatizing agent with a fixed charge (e.g., TMPP or Girard's T). We found, as anticipated, that the total amount of reagent required for derivatization on tissue was significantly greater than for an equivalent amount of analyte in solution. This is due to the 
presence of many additional endogenous reactive sites in tissue. Precoating the MALDI target allows the necessary amount of reagent to be homogenously deposited while minimizing analyte delocalization.

\section{Acknowledgments}

The authors acknowledge support for this work (in part) by the Bill and Melinda Gates Foundation Accelerator grant N01 HD23342, (in part) by NIH/NIGMS grant 5R01 GM58008, and (in part) by the Intramural Research Program of NIAID, NIH. The assistance of Dr. Joey C. Latham, Dr. Kenneth E. Schriver, and Jamie Allen (Vanderbilt) and Danielle Weiner and Jacqueline Gonzales (NIH) is gratefully acknowledged.

\section{References}

1. Caprioli, R.M., Farmer, T.B., Gile, J.: Molecular imaging of biological samples: localization of peptides and proteins using MALDI-TOF MS. Anal. Chem. 69(23), 4751-4760 (1997)

2. Stoeckli, M., Chaurand, P., Hallahan, D.E., Caprioli, R.M.: Imaging mass spectrometry: A new technology for the analysis of protein expression in mammalian tissues. Nat. Med. 7(4), 493-496 (2001)

3. Chaurand, P., Sanders, M.E., Jensen, R.A., Caprioli, R.M.: Proteomics in diagnostic pathology: profiling and imaging proteins directly in tissue sections. Am. J. Pathol. 165(4), 1057-1068 (2004)

4. Burnum, K.E., et al.: Imaging mass spectrometry reveals unique protein profiles during embryo implantation. Endocrinology 149(7), 3274-3278 (2008)

5. Stoeckli, M., Staab, D., Staufenbiel, M., Wiederhold, K.-H., Signor, L.: Molecular imaging of amyloid $\beta$ peptides in mouse brain sections using mass spectrometry. Anal. Biochem. 311, 33-39 (2002)

6. Andersson, M., Groseclose, M.R., Deutch, A.Y., Caprioli, R.M.: Imaging mass spectrometry of proteins and peptides: 3D volume reconstruction. Nat. Methods 5(1), 101-108 (2008)

7. Hankin, J.A., Barkley, R.M., Murphy, R.C.: Sublimation as a method of matrix application for mass spectrometric imaging. J. Am. Soc. Mass Spectrom. 18(9), 1646-1652 (2007)

8. Burnum, K.E., et al.: Spatial and temporal alterations of phospholipids determined by mass spectrometry during mouse embryo implantation. $J$. Lipid Res. 50(11), 2290- 2298 (2009)

9. Garrett, T.J., Yost, R.A.: Analysis of intact tissue by intermediatepressure MALDI on a linear ion trap mass spectrometer. Anal. Chem. 78(7), 2465-2469 (2006)

10. Troendle, F.J., Reddick, C.D., Yost, R.A.: Detection of pharmaceutical compounds in tissue by matrix-assisted laser desorption/ionization and laser desorption/chemical ionization tandem mass spectrometry with a quadrupole ion trap. J. Am. Soc. Mass Spectrom. 10(12), 1315-1321 (1999)

11. Reyzer, M.L., Hsieh, Y., Ng, K., Korfmacher, W.A., Caprioli, R.M.: Direct analysis of drug candidates in tissue by matrix-assisted laser desorption/ionization mass spectrometry. J. Mass Spectrom. 38, 10811092 (2003)

12. Khatib-Shahidi, S., Andersson, M., Herman, J.L., Gillespie, T.A., Caprioli, R.M.: Direct molecular analysis of whole-body animal tissue sections by imaging MALDI mass spectrometry. Anal. Chem. 78(18), 6448-6456 (2006)

13. Seeley, E.H., Oppenheimer, S.R., Mi, D., Chaurand, P., Caprioli, R.M.: Enhancement of protein sensitivity for MALDI imaging mass spectrometry after chemical treatment of tissue sections. J. Am. Soc. Mass Spectrom. 19(8), 1069-1077 (2008)

14. Grey, A.C., Chaurand, P., Caprioli, R.M., Schey, K.L.: MALDI imaging mass spectrometry of integral membrane proteins from ocular lens and retinal tissue. J. Proteome Res. 8(7), 3278-3283 (2009)

15. Chaurand, P., Norris, J.L., Cornett, D.S., Mobley, J.A., Caprioli, R.M.: New developments in profiling and imaging of proteins from tissue sections by MALDI mass spectrometry. J. Proteome Res. 5(11), 2889 2900 (2006)
16. Lemaire, R., et al.: MALDI-MS direct tissue analysis of proteins: Improving signal sensitivity using organic treatments. Anal. Chem. 78 (20), 7145-7153 (2006)

17. Wang, H.-Y. J., Jackson, S.N., McEuen, J., Woods, A.S.: Localization and analyses of small drug molecules in rat brain tissue sections. Anal. Chem. 77(20), 6682-6686 (2005)

18. Cornett, D.S., Frappier, S.L., Caprioli, R.M.: MALDI-FTICR imaging mass spectrometry of drugs and metabolites in tissue. Anal. Chem. 80 (14), 5648-5653 (2008)

19. Blau, K., King, G.S. (eds.) Handbook of Derivatives for Chromatography. Heyden and Son, Ltd.: London, p. 576 (1977)

20. Knapp, D.R. (ed.) Handbook of Analytical Derivatization Reactions. John Wiley and Sons, Inc.: New York, p. 741 (1979)

21. Liu, X., Qiu, H., Lee, R.K., Chen, W., Li, J.: Methylamidation for sialoglycomics by MALDI-MS: A facile derivatization strategy for both a2,3- and a2,6-linked sialic acids. Anal Chem. 82(19), 8300-8306 (2010)

22. Rohmer, M., et al.: 3-aminoquinoline acting as matrix and derivatizing agent for MALDI MS analysis of oligosaccharides. Anal. Chem. 82(9), 3719-3726 (2010)

23. Lin, C., Hung, W.T., Chen, C.H., Fang, J.M., Yang, W.B.: A new naphthimidazole derivative for saccharide labeling with enhanced sensitivity in mass spectrometry detection. Rapid Commun. Mass Spectrom. 24, 85-94 (2010)

24. Xie, J., et al.: Extraction and derivatization in single drop coupled to MALDI-FTICR-MS for selective determination of small molecule aldehydes in single puff smoke. Anal. Chim. Acta. 638(2), 198-201 (2009)

25. Durairaj, A., Limbach, P.A.: Matrix-assisted laser desorption/ionization mass spectrometry screening for pseudouridine in mixtures of small RNAs by chemical derivatization, RNase digestion and signature products. Rapid Commun. Mass Spectrom. 22, 3727-3734 (2008)

26. Li, J., et al.: Enhanced detection of thiol peptides by matrix-assisted laser desorption/ionization mass spectrometry after selective derivatization with a tailor-made quaternary ammonium tag containing maleimidyl group. Rapid Commun. Mass Spectrom. 21, 2608-2612 (2007)

27. Snovida, S.I., Chen, V.C., Perreault, H.: Use of a 2,5-dihydroxybenzoic acid/aniline MALDI matrix for improved detection and on-target derivatization of glycans: a preliminary report. Anal. Chem. 78(24), 8561-8568 (2006)

28. Ullmer, R., Plematl, A., Rizzi, A.: Derivatization by 6-aminoquinolyl$\mathrm{N}$-hydroxysuccinimidyl carbamate for enhancing the ionization yield of small peptides and glycopeptides in matrix-assisted laser desorption/ ionization and electrospray ionization mass spectrometry. Rapid Commun. Mass Spectrom. 20, 1469-1479 (2006)

29. Khan, M.A., et al.: Analysis of derivatised steroids by matrix-assisted laser desorption/ionisation and post-source decay mass spectrometry. Steroids 71(1), 42-53 (2006)

30. Sekiya, S., Wada, Y., Tanaka, K.: Derivatization for stabilizing sialic acids in MALDI-MS. Anal Chem. 77(15), 4962-4968 (2005)

31. Franck, J.; El Ayed, M., Wisztorski, M., Salzet, M., Fournier, I.: Ontissue N-terminal peptide derivatizations for enhancing protein identification in MALDI mass spectrometric imaging strategies. Anal. Chem. 81(20), 8305-8317 (2009)

32. Vorm, O., Roepstorff, P., Mann, M.: Improved resolution and very high sensitivity in MALDI TOF of matrix surfaces made by fast evaporation. Anal Chem. 66(19), 3281-3287 (1994)

33. Zhang, H., Caprioli, R.M.: Capillary electrophoresis combined with matrix-assisted laser desorption/ionization mass spectrometry; continuous sample deposition on a matrix-precoated membrane target. J. Mass Spectrom. 31(9), 1039-1046 (1996)

34. Wall, D. B., et al.: Continuous sample deposition from reversed-phase liquid chromatography to tracks on a matrix-assisted laser desorption/ ionization precoated target for the analysis of protein digests. Electrophoresis 23(18), 3193-3204 (2002)

35. Grove, K., Frappier, S., Caprioli, R.: Matrix precoated MALDI MS targets for small molecule imaging in tissues. J. Am. Soc. Mass Spectrom. 22(1), 192-195 (2011)

36. Peloquin, C.A.: Pharmacology of the antimycobacterial drugs. Med. Clin. North Am. 77(6), 1253-1262 (1993)

37. Jindani, A., Dore, C.J., Mitchison, D.A.; Bactericidal and sterilizing activities of antituberculosis drugs during the first 14 days. Am. J. Respir. Crit. Care Med. 167(10), 1348-1354 (2003)

38. Mitchison, D.A.: The search for new sterilizing anti-tuberculosis drugs. Front Biosci. 9, 1059-1072 (2004) 
39. Young, D.B., Perkins, M.D., Duncan, K., Barry, C.E. III.: Confronting the scientific obstacles to global control of tuberculosis. J. Clin. Invest. 118(4), 1255-1265 (2008)

40. Seifart, H.I., Gent, W.L., Parkin, D.P., van Jaarsveld, P.P., Donald, P. R.: High-performance liquid chromatographic determination of isoniazid, acetylisoniazid and hydrazine in biological fluids. J. Chromatogr. B 674(2), 269-275 (1995)

41. Kim, M., Stewart, J.T.: HPLC post-column derivatization of aromatic amines using N-methyl-9-chloroacridinium triflate. Microchim. Acta 102(4), 221-232 (1990)

42. Driouich, R., Takayanagi, T., Mitsuko, O., Motomizu, S.: Investigation of salicylaldehyde-5-sulfonate as a precolumn derivatizing agent for the determination of $\mathrm{n}$-alkane diamines, lysine, diaminopimelic acid, and isoniazid by capillary zone electrophoresis. J. Pharmaceut. Biomed. Anal. 30(5), 1523-1530 (2003)

43. Horikawa, R., Tanimura, T., Tamura, Z.: Method for fluorescence detection in the high-performance liquid chromatography of $84-3$ ketosteroids. J. Chromatogr A 168(2), 526-529 (1979)

44. Cohen, S.A., Michaud, D.P.: Synthesis of a fluorescent derivatizing reagent, 6-aminoquinolyl-N-hydroxysuccinimidyl carbamate, and its application for the analysis of hydrolysate amino acids via high-performance liquid chromatography. Anal. Biochem. 211(2), 279-287 (1993)

45. Huang, Z.-H., Shen, T., Wu, J., Gage, D.A., Watson, J.T.: Protein sequencing by matrix-assisted laser desorption ionization-postsource decay-mass spectrometry analysis of theN-Tris(2,4,6-trimethoxyphenyl) phosphine-acetylated tryptic digests. Anal. Biochem. 268(2), 305-317 (1999)
46. Via, L.E., et al.: Tuberculous granulomas are hypoxic in guinea pigs, rabbits, and nonhuman primates. Infect. Immun. 76(6), 2333-2340 (2008)

47. Fang, P.-F., et al.: Simultaneous determination of isoniazid, rifampicin, levofloxacin in mouse tissues and plasma by high performance liquid chromatography-tandem mass spectrometry. J. Chromatogr. B 878(24), 2286-2291 (2010)

48. Wang, A., et al.: HPLC-MS analysis of isoniazid in dog plasma. Chromatographia 66(9), 741-745 (2007)

49. Ng, K.-Y., et al.: Quantification of isoniazid and acetylisoniazid in rat plasma and alveolar macrophages by liquid chromatography-tandem mass spectrometry with on-line extraction. J. Chromatogr. B 847(2), 188-198 (2007)

50. Song, S. H., et al.: Simultaneous determination of first-line antituberculosis drugs and their major metabolic ratios by liquid chromatography/tandem mass spectrometry. Rapid Commun. Mass Spectrom. 21(7), 1331-1338 (2007)

51. Huang, L., et al.: Development and validation of a hydrophilic interaction liquid chromatography-tandem mass spectrometry method for determination of isoniazid in human plasma. J. Chromatogr. B 877 (3), 285-290 (2009)

52. Pound, A.W., Pound, J.R.: The oxidation of cinnamaldehyde. J. Phys. Chem. 38, 1045-1049 (1934)

53. Reich, R.F., Cudzilo, K., Levisky, J.A., Yost, R.A.: Quantitative MALDI-MS $(n)$ analysis of cocaine in the autopsied brain of a human cocaine user employing a wide isolation window and internal standards. J. Am. Soc. Mass Spectrom. 21(4), 564- 571 (2010) 